\title{
Theoretical studies of muon capture on light nuclei
}

\author{
Laura E. Marcucci* \\ Dipartimento di Fisica "E. Fermi", Università di Pisa \\ INFN, Sezione di Pisa \\ I-56127 Pisa, Italy \\ E-mail: laura.marcucciedf.unipi.it
}

\begin{abstract}
We review the present status of theoretical studies of muon capture reactions on light nuclei. In particular we consider the two reactions ${ }^{2} \mathrm{H}\left(\mu^{-}, v_{\mu}\right) n n$ and ${ }^{3} \mathrm{He}\left(\mu^{-}, v_{\mu}\right)^{3} \mathrm{H}$, and the most recent calculations performed within the chiral effective field theory framework. The unresolved discrepancies among the different calculations and future developments are also discussed.
\end{abstract}

The 7th International Workshop on Chiral Dynamics,

August 6-10, 2012

Jefferson Lab, Newport News, Virginia, USA

*Speaker. 


\section{Introduction}

In the past few years there has been a significant body of theoretical work on muon captures on light nuclei, in particular on the reactions

$$
\begin{aligned}
& \mu^{-}+d \rightarrow n+n+v_{\mu}, \\
& \mu^{-}+{ }^{3} \mathrm{He} \rightarrow{ }^{3} \mathrm{H}+v_{\mu} .
\end{aligned}
$$

This large interest is motivated by the fact that these processes are related to astrophysically relevant reactions, such as the weak captures on proton and ${ }^{3} \mathrm{He}$ (the $p p$ and hep reactions), whose rates cannot be measured experimentally [1].

The observables of interest for the two reactions are the doublet capture rate $\Gamma^{D}$ for reaction (1.1), i.e., the rate obtained when the stopped muons are captured from the initial doublet hyperfine state, and the total capture rate $\Gamma_{0}$ for reaction (1.2). The experimental situation for reaction (1.2) is quite clear: a very precise determination yielded $\Gamma_{0}=(1496 \pm 4) \sec ^{-1}$ [2], a value consistent with those of earlier measurements, although these were affected by considerably larger uncertainties. On the other hand, $\Gamma^{D}$ is poorly known: the available experimental data are (365 \pm 96$) \mathrm{sec}^{-1}$ [3], $(445 \pm 60) \mathrm{sec}^{-1}$ [4], $(470 \pm 29) \mathrm{sec}^{-1}$ [5] and (409 \pm 40$) \mathrm{sec}^{-1}$ [6]. These measurements, while consistent with each other, are not very precise, with errors in the $6-10$ $\%$ range. However, there is hope to have this situation clarified by the MuSun Collaboration [7], which is performing at present an experiment at the Paul Scherrer Institut, with the goal of measuring $\Gamma^{D}$ with a precision of $1.5 \%$. Part of the renewed interest on the capture processes (1.1) and (1.2) has been indeed spurred by this experiment.

Theoretical work on reactions (1.1) and (1.2) is very extensive. The status ante 2012 has been recently reviewed in Ref. [8], where the theoretical formalism and the details of the different calculations are clearly discussed. Here, we briefly review again only the latest work of Refs. [9, 10].

In Refs. [9, 10], reactions (1.1) and (1.2) are simultaneously studied within two different schemes: the "potential model approach" (PMA), also known as "standard nuclear physics approach", and the approach known as the "hybrid" chiral effective field theory $(\chi \mathrm{EFT})$, often indicated with $\chi \mathrm{EFT}^{*}$. In PMA, Hamiltonians based on conventional two-nucleon (NN) and threenucleon (NNN) potentials are used to calculate the nuclear wave functions, and the weak transition operator includes, beyond the one-body contribution (the impulse approximation-IA) associated with the basic process $p+\mu^{-} \rightarrow n+v_{\mu}$, meson-exchange currents as well as currents arising from the excitation of $\Delta$-isobar degrees of freedom [11]. In the $\chi \mathrm{EFT}^{*}$ approach, the weak operators are derived in $\chi \mathrm{EFT}$, but their matrix elements are evaluated between wave functions obtained from conventional potentials. Typically, the PMA and $\chi \mathrm{EFT}^{*}$ predictions are in good agreement with each other. For example, the PMA and $\chi$ EFT* calculations for $\Gamma^{D}$ give $391 \mathrm{sec}^{-1}$ and $(393 \pm 1) \mathrm{sec}^{-1}$, respectively [9]. For $\Gamma_{0}$, the PMA and $\chi \mathrm{EFT}^{*}$ calculations give $1486 \mathrm{sec}^{-1}$ and $(1484 \pm 4) \mathrm{sec}^{-1}$, respectively. Note that in Ref. [9] the radiative corrections of Ref. [12] were not included. These would increase the PMA ( $\chi$ EFT*) central values of Ref. [9] to 392 (395) $\mathrm{sec}^{-1}$ for reaction (1.1), and 1496 (1494) $\mathrm{sec}^{-1}$ for reaction (1.2). In summary, the combined PMA and

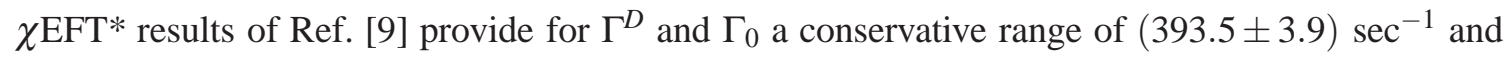
$(1493 \pm 19) \mathrm{sec}^{-1}$, when radiative corrections with their uncertainties are considered [12]. 
Note that just one year before the work of Refs. $[9,10]$, an other calculation of $\Gamma^{D}$ was performed in Ref. [13] within the PMA, although the meson-exchange currents were of different origin. The results of Ref. [13] differ from those of Refs. $[9,10]$ by $7-10 \%$. Only very recently, the origin of this discrepancy has been understood, as it will discussed in the next section.

The present paper continues as follows: in Sec. 2 we review and discuss the results of the first "non-hybrid" $\chi$ EFT calculation of reactions (1.1) and (1.2). Some concluding remarks are given in Sec. 3 .

\section{The "non-hybrid" $\chi$ EFT study of muon capture}

The first "non-hybrid" $\chi$ EFT study of reactions (1.1) and (1.2) has been performed in Ref. [14]. The nuclear interaction consists of NN and NNN potentials. The NN potential has been derived in Refs. $[15,16]$ up to next-to-next-to-next-to leading order (N3LO). It consists of one-pion- and two-pion-exchange and of contact terms. The low-energy constants (LEC's) entering the contact terms have been constrained by accurate fits to the NN scattering database at energies below the pion production threshold. The NNN potential, which first contributes at next-to-next-to leading order (N2LO), includes $S$ - and $P$-wave two-pion-exchange - its $P$-wave piece is the familiar FujitaMiyazawa NNN potential-a one-pion-exchange plus NN contact term with LEC $c_{D}$ and a NNN contact terms with LEC $c_{E}$. The N2LO NNN potential used in Ref. [14] is the local version obtained in Ref. [17].

The vector part of the weak current has been derived in $\chi$ EFT up to N3LO by several groups. However, only in Refs. [18] and [19] a consistent model for both the vector and axial pieces of the weak current has been constructed up to N3LO. Therefore, this model has been considered in Ref. [14]. In this model, the one-body operators are the same as those obtained in the PMA by retaining, in the expansion of the covariant single-nucleon four-current, corrections up to order $(v / c)^{2}$ relative to the leading-order term [11]. The two-body operators in the axial current include a one-pion-exchange contribution, involving the known LEC's $c_{3}$ and $c_{4}$ (determined by fits to the NN data [16]), and one contact current, whose strength is parametrized by the LEC $d_{R}$ (see below). In the axial charge, only one-pion-exchange contributes, and the associated operator is proportional to $g_{A} / F_{\pi}^{2}$, where $g_{A}\left(F_{\pi}\right)$ is the single-nucleon axial coupling constant (pion decay constant). The vector weak current is related (via the CVC constraint) to the electromagnetic current, which includes at N3LO one-pion- and two-pion-exchange (i.e., one-loop corrections), as well as isoscalar and isovector contact terms, whose strengths are parametrized by the LEC's denoted, respectively, as $g_{4 S}$ and $g_{4 V}[18,19]$. No two-nucleon vector charge operators are present at N3LO. Finally, we notice that due to the power-law behavior for large momenta of both potentials and currents, these have been regularized by introducing a momentum-cutoff function, with cutoff $\Lambda$ taken to be $500 \mathrm{MeV}$ and $600 \mathrm{MeV}$.

The mentioned LEC's $d_{R}, c_{D}, c_{E}, g_{4 S}$, and $g_{4 V}$ have been determined as follows: as it has been observed in Refs. [20] and [21], the LEC's $d_{R}$ and $c_{D}$ are related to each other via the relation

$$
d_{R}=\frac{M_{N}}{\Lambda_{\chi} g_{A}} c_{D}+\frac{1}{3} M_{N}\left(c_{3}+2 c_{4}\right)+\frac{1}{6},
$$

where $M_{N}$ is the nucleon mass and $\Lambda_{\chi}=700 \mathrm{MeV}$ is the the chiral-symmetry-breaking scale. Then, the calculation is implemented as follows. The ${ }^{3} \mathrm{H}$ and ${ }^{3} \mathrm{He}$ ground state wave functions are 
calculated with the hyperspherical-harmonics method (see Ref. [22] for a review) using the chiral $\mathrm{NN}+\mathrm{NNN}$ potentials of Refs. [15, 16, 17] for $\Lambda=500$ and $600 \mathrm{MeV}$. The corresponding set of LEC's $\left\{c_{D}, c_{E}\right\}$ is determined by fitting the $A=3$ experimental binding energies, $\mathrm{BE}\left({ }^{3} \mathrm{H}\right)=8.475$ $\mathrm{MeV}$ and $\mathrm{BE}\left({ }^{3} \mathrm{He}\right)=7.725 \mathrm{MeV}$, corrected for small contributions $\left(+7 \mathrm{keV}\right.$ in ${ }^{3} \mathrm{H}$ and $-7 \mathrm{keV}$ in $\left.{ }^{3} \mathrm{He}\right)$ due to the $n$ - $p$ mass difference [23]. Then the range $c_{D} \in[-3,3]$ is considered, and, in correspondence to each $c_{D}$ in this range, $c_{E}$ is determined so as to reproduce either $\mathrm{BE}\left({ }^{3} \mathrm{H}\right)$ or $\mathrm{BE}\left({ }^{3} \mathrm{He}\right)$. The resulting trajectories, shown in Fig. 1 of Ref. [14], are nearly indistinguishable. Then, for each set of $\left\{c_{D}, c_{E}\right\}$, the triton and ${ }^{3} \mathrm{He}$ wave functions are calculated and, using the $\chi$ EFT weak axial current discussed above, the Gamow-Teller matrix element of tritium $\beta$-decay $\left(\mathrm{GT}^{\mathrm{TH}}\right)$ is determined. The ratio $\mathrm{GT}^{\mathrm{TH}} / \mathrm{GT}^{\mathrm{EXP}}$ is considered, for both $\Lambda=500$ and $600 \mathrm{MeV}$, with $\mathrm{GT}^{\mathrm{EXP}}=0.955 \pm 0.004[9,14]$, and the range of $c_{D}$ values, for which $\mathrm{GT}^{\mathrm{TH}}=\mathrm{GT}^{\mathrm{EXP}}$ within the experimental error, is found to be $[-0.20,-0.04]$ for $\Lambda=500 \mathrm{MeV}$, and $[-0.32,-0.19]$ for $\Lambda=600$ $\mathrm{MeV}$. The corresponding ranges for $c_{E}$ are $[-0.208,-0.184]$ and $[-0.857,-0.833]$, respectively.

For the minimum and maximum values of $\left\{c_{D}, c_{E}\right\}$ in the selected range, i.e., $\{-0.20,-0.208\}$ and $\{-0.04,-0.184\}$ for $\Lambda=500 \mathrm{MeV}$, and $\{-0.32,-0.857\}$ and $\{-0.19,-0.833\}$ for $\Lambda=600$ $\mathrm{MeV}$, the isoscalar and isovector LEC's, $g_{4 S}$ and $g_{4 V}$, entering the NN contact terms of the electromagnetic current, are determined by reproducing the $A=3$ magnetic moments. These LEC's are listed in Table I of Ref. [14]. At this point, the potential and current models are fully constrained, and the results for $\Gamma^{D}$ and $\Gamma_{0}$ are $\chi$ EFT predictions. They are found to be $\Gamma^{D}=(399 \pm 3) \mathrm{sec}^{-1}$ and $\Gamma_{0}=(1494 \pm 21) \mathrm{sec}^{-1}$, including electroweak radiative corrections [12]. These results are in good agreement with those of Refs. [9, 10], as well as with experimental data, but not with those of Ref. [13], for which the discrepancy remains of the order of 4-9\%. We now discuss in some detail the origin of this discrepancy. Some of the authors of Ref. [13] have performed in Ref. [24] a $\chi$ EFT calculation of $\Gamma^{D}$ very similar to the one presented in this section and in fact contemporary to it. In this calculation, $\Gamma^{D}$ has been found in the range $383.8-392.4 \mathrm{sec}^{-1}$, depending on the $\chi$ EFT two-nucleon potential used. This result is very different from the previous PMA calculation of Ref. [13], and it is in much better agreement with the results of Refs. [9, 10, 14]. In fact, the study of Ref. [24] has a very interesting history, according to the two different versions of the manuscript, still present on the archive, www.arXiv. org. In the very first version of the manuscript, $\Gamma^{D}$ was found in the range $401.2-409.8 \mathrm{sec}^{-1}$, in good agreement with the results of Ref. [13], and again in disagreement with those of Refs. [9, 10, 14]. The printed version of the manuscript, which corresponds to the second version of the preprint on the archive, however, reported the range quoted above, $383.8-392.4 \mathrm{sec}^{-1}$. Therefore, we can conclude that one or more computational errors were found before the submission of the second version, suggesting that very likely the results of Ref. [13] were also affected by the same errors. For this reason, they should be disregarded. Finally, it should be pointed out that the authors of Ref. [24] consider, besides the $\chi$ EFT potential mentioned here of Ref. [15], also two other potentials, labelled $\operatorname{EGM(204)}$ and $\operatorname{EGM}(205)$, taken from Ref. [25]. The quoted range $383.8-392.4 \mathrm{sec}^{-1}$ is obtained excluding the $\operatorname{EGM}(205)$ results. Such range would become $383.8-419.1 \mathrm{sec}^{-1}$ when all the $\chi$ EFT potential models are considered, with a theoretical uncertainty of about $8 \%$. The authors, though, do not provide any explanation on why the $\operatorname{EGM}(205)$ results should be excluded. Such a large theoretical uncertainty, as argued by the authors themselves, is presumably due to the fact that the $c_{D}-c_{E}$ fitting procedure is not always applied including the NNN potential. In any case, the results of 
Ref. [24] and the ones discussed here $[9,10,14]$ are in agreement within few $\%$.

\section{Conclusions}

The most recent theoretical studies of the muon capture reactions on deuteron and ${ }^{3} \mathrm{He}$ have been reviewed and discussed. They are performed either using phenomenological potentials and currents, or phenomenological potentials in conjunction with weak currents derived in $\chi$ EFT, or finally using both potentials and currents obtained consistently within the $\chi$ EFT approach. The calculated values for the doublet capture rate (total capture rate) for muon capture on deuteron $\left({ }^{3} \mathrm{He}\right)$ are in agreement with each other and in agreement with the available experimental data. Furthermore, the theoretical uncertainty is reduced to the level of a \%, even for the latest "nonhybrid" $\chi$ EFT calculation. The differences between the calculations reviewed here and those of Refs. [13, 24] have been discussed. In particular, we have pointed out that the results of Ref. [13] were clearly affected by some computational errors and should be disregarded. We expect the authors of Ref. [13] to publish one day an erratum, eventually.

We would like to conclude with the following observations: (i) the present "non-hybrid" $\chi \mathrm{EFT}$ framework can be used to calculate reactions of astrophysical interest, as the $p p$ or hep reactions, as well as the proton-deuteron radiative capture reaction. Work along this line is strongly pursued. (ii) Some small inconsistencies are still present in what we have called "non-hybrid" $\chi$ EFT framework. In fact, the NN and NNN potentials are derived at two different chiral order (N3LO and N2LO, respectively), and the momentum-cutoff functions used for the NNN potential and nuclear currents are different than those present in the NN potential. However, if these inconsistencies were significant, we would have expected, as a general trend of $\chi$ EFT calculations, a strong cutoff dependence of the results. Instead, the cutoff-dependence of the presented results is extremely weak.

\section{References}

[1] E.G. Adelberger et al., Solar fusion cross sections II: the pp chain and CNO cycles, Rev. Mod. Phys. 83 (2011) 195 [arXiv: 1004.2318 ].

[2] P. Ackerbauer et al., A precision measurement of nuclear muon capture on ${ }^{3} \mathrm{He}$, Phys. Lett. B 417 (1998) 224.

[3] I-T. Wang et al. Muon capture in $(p \mu d)^{+}$molecules, Phys. Rev. 139 (1965) B1528.

[4] A. Bertin A, Muon-capture in gaseous deuterium, Phys. Rev. D 8 (1973) 3774.

[5] G. Bardin et al., A measurement of the muon-capture rate in liquid deuterium by the lifetime technique Nucl. Phys. A 453 (1986) 591.

[6] M. Cargnelli M et al., in proceedings of Workshop on fundamental $\mu$ physics, (Los Alamos, LA 10714C) (1986); in proceedings of Nuclear Weak Process and Nuclear Structure, Yamada Conference XXIII, ed. M. Morita, H. Ejiri, H. Ohtsubo, and T. Sato (Word Scientific, Singapore), p. 115 (1989).

[7] V.A. Andreev et al. [MuSun Collaboration], Muon Capture on the Deuteron - The MuSun Experiment, arXiv:1004.1754.

[8] L.E. Marcucci, Muon Capture on Deuteron and ${ }^{3} H e$ : A Personal Review, Int. J. Mod. Phys. A 27 (2012) 1230006 [arXiv:1112.0113]. 
[9] L.E. Marcucci et al., Muon capture on deuteron and ${ }^{3}$ He, Phys. Rev. C 83 (2011) 014002 [arXiv: 1008.1172$]$.

[10] L.E. Marcucci and M. Piarulli, Muon capture on light nuclei, Few-Body Syst. 49 (2011) 35 [arXiv:1009.4016].

[11] L.E. Marcucci et al., Weak proton capture on ${ }^{3} H e$, Phys. Rev. C 63 (2000) 015801 [arXiv:nuc-th/0006005].

[12] A. Czarnecki, W.J. Marciano, and A. Sirlin, Electroweak radiative corrections to muon capture, Phys. Rev. Lett. 99 (2007) 032003.

[13] P. Ricci, E. Truhlík, B. Mosconi, and J. Smejkal, Muon Capture in Deuterium, Nucl. Phys. A 837 (2010) 110 [arXiv:0912.1989].

[14] L.E. Marcucci et al., Chiral effective field theory predictions for muon capture on deuteron and ${ }^{3} \mathrm{He}$, Phy. Rev. Lett. 108 (2012) 052502 [arXiv:1109.5563].

[15] D.R. Entem and R. Machleidt, Accurate charge-dependent nucleon-nucleon potential at fourth order of chiral perturbation theory, Phys. Rev. C 68 (2003) 041001 [arXiv: nucl-th/ 0304018 ].

[16] R. Machleidt and D.R. Entem, Chiral effective field theory and nuclear forces, Phys. Rep. 503 (2011) 1 [arXiv:1105.2919].

[17] P. Navrátil, Local three-nucleon interaction from chiral effective field theory, Few-Body Syst. 41 (2007) 117 [arXiv:0707.4680].

[18] T.-S. Park, D.-P. Min, and M. Rho, Chiral lagrangian approach to exchange vector currents in nuclei, Nucl. Phys. A 596 (1996) 515 [arXiv: nucl-th/9505017]; Y.-H. Song, R. Lazauskas, and T.-S. Park, Up to N3LO heavy-baryon chiral perturbation theory calculation for the M1 properties of three-nucleon systems, Phys. Rev. C 79 (2009) 064002.

[19] T.-S. Park et al., Parameter-free effective field theory calculation for the solar proton-fusion and hep processes, Phys. Rev. C 67 (2003) 055206 [arXiv:nucl-th/0208055].

[20] A. Gardestig and D.R. Phillips, How low-energy weak reactions can constrain three-nucleon forces and the neutron-neutron scattering length, Phys. Rev. Lett. 96 (2006) 232301 [arXiv:nucl-th/0603045].

[21] D. Gazit, S. Quaglioni, and P. Navrátil Three-nucleon low-energy constants from the consistency of interactions and currents in chiral effective field theory, Phys. Rev. Lett. 103 (2009) 102502 [arXiv:0812.4444].

[22] A. Kievsky et al., A high-precision variational approach to three- and four-nucleon bound and zero-energy scattering states, J. Phys. G: Nucl. Part. Phys. 35 (2008) 063101 [arXiv:0805.4688].

[23] A. Nogga et al., Three-nucleon bound states using realistic potential models, Phys. Rev. C 67 (2003) 034004 [arXiv:nucl-th/0202037].

[24] J. Adam, M. Tater, E. Truhlík, E. Epelbaum, R. Machleidt, and P. Ricci, Calculation of Doublet Capture Rate for Muon Capture in Deuterium within Chiral Effective Field Theory, Phys. Lett. B 709 (2012) 93 [arXiv:1110.3183].

[25] E. Epelbaum, W. Glöckle, and U.-G. Meissner, The two-nucleon system at next-to-next-to-next-to-leading order, Nucl. Phys. A 747 (2005) 362 [arXiv: nucl-th/ 0405048 ]. 